\title{
Q-Space Analysis of Light Scattering by Gaussian Random Spheres
}

Justin B. Maughan, Christopher M. Sorensen, Amitabha Chakrabarti*

Physics Department, 116 Cardwell Hall, 1228 N. 17th St, Manhattan KS 66506-2601

* Corresponding author. E-mail address: amitc@phys.ksu.edu (A. Chakrabarti)

\begin{abstract}
Q-space analysis is applied to the scattered intensity from Gaussian Random Spheres (GRSs). Q-space analysis involves plotting the scattered intensity vs. the magnitude of the scattering wave vector $q=2 k \sin \theta / 2$ where $k=2 \pi / \lambda$ with $\lambda$ the wavelength and $\theta$ is the scattering angle, on a $\log -\log$ plot. The light scattering properties of GRSs were calculated using a discrete dipole approximation algorithm. The GRSs had $\sigma=0.2$ and $v=3$, where $\sigma$ is the relative standard deviation in the radial direction, and $v$ describes fluctuations in the angular direction. Calculations were systematically performed with size parameters ranging from 10 to 30 , and with a relative index of refraction, $\mathrm{m}$, ranging from 1.01 to 1.5. The results show quantifiable, power law descriptions of the scattered intensity and a Rayleigh functionality of the forward scattered intensity that depend upon the phase shift parameter $\rho=2 k R_{e q}|m-1|$ where $R_{e q}$ is an equivalent radius. Similar functionalities have been observed in both spheres and other irregularly shaped particles. These results continue the implication that there is a comprehensive description of light scattering for all particles that can be uncovered with the application of Q-space.
\end{abstract}

Keywords:

Light scattering

Gaussian Random Sphere

Q-space

Phase shift parameter

\section{Introduction}

A topic of current interest in the theory of light scattering is how irregularly shaped particles scatter light. This problem is not only of intellectual importance but also plays a role in climate models as most aerosols in the atmosphere are irregular. Some of the sources of these particles are, but not limited to, mineral dusts, volcanic ash, soot, and snow and ice crystals that display a wide variety of irregular shapes. Computational advancements over the last two decades have made theoretical studies of scattering from irregular shapes more practical. However, the results, although useful, yield phase functions that are not amenable to quantitative description or differentiation for different shapes. Thus there is a 
strong need for another point of view for scattering that will allow for such . quantitative descriptions. .

In the recent past we have developed a unique approach, Q-space analysis . (see below), which provides quantitative descriptions of the angular patterns of. scattering by particles. Q-space analysis has been applied to both experimental and . numerical (via the Mie solution) results of scattering by spheres [1-4]. In addition, . application of this analysis to scattering from various aggregates [5,6], dust. particles, and small irregularly shaped perturbed spheres $[7,8]$ has been published . in the literature. The application of Q-space to these various shapes has uncovered. common quantifiable behaviors in the light scattering (see below)[9]. .

The Gaussian Random Sphere (GRS) model is based on correlated. fluctuations around an underlying spherical shape and has been used to model a . wide range of irregularly shaped particles from the macroscopic size to the. microscopic. Types of particles previously modeled by GRS include Saharan dust. particles [10,11], feldspar, and ice crystals [12,13]. The widespread and relevant. applicability of GRS makes it an ideal candidate for searching for universal patterns . in light scattering when Q-space analysis is applied. .

In this paper, we apply Q-space analysis to light scattering intensity from . Gaussian Random Spheres. The light scattering properties of GRSs were calculated . using a discrete dipole approximation algorithm. We present results by . systematically varying the size parameter over a large range. Similar to both spheres . and other irregularly shaped particles, our results for GRSs show power law. descriptions of the scattered intensity and a Rayleigh functionality of the forward . scattered intensity that depend upon the phase shift parameter. .

\section{Brief Summary of Q-space Analysis}

Q-space analysis involves plotting the scattered intensity vs. the magnitude . of the scattering wave vector $q$.

$\dot{q}=2 k \sin (\theta / 2)$

on a log-log plot [9]. Here $k=2 \pi / \lambda$ with $\lambda$ is the wavelength and $\theta$ is the scattering . angle. Much insight into universal properties in scattering is gained by plotting the . scattered intensity vs. a unit-less quantity $q R_{e q}$ where $R_{e q}$ is some "equivalent". radius such as the radius of gyration $R_{g}$ for aggregates, the volume equivalent radius .

$R_{\text {veq }}$ for 3D irregular shapes, or simply the radius $R$ for spheres. When Q-space. analysis is applied, patterns and structures in the scattered intensities have been . found for a wide variety of particle shapes that are not apparent otherwise [1-9]. .

Past results of Q-space analysis can be summarized as a general semi-. quantitative description of scattering for any particle shape in the following way: . 
1. $q R_{e q}<1$ The Forward Scattering Lobe which is independent of $q$.

2. $q R_{e q} \sim 1$ The Guinier[14] regime where functionality with $q$ (hence $\theta$ ) begins.

3. $2<q R_{e q}<5$ The Dip in the light scattering seems to be a common phenomenon in many shapes.

4. $q R_{e q}>1$ The Power Law regime (the Dip not withstanding). Here the scattering follows power laws such that $\mathrm{I} \sim\left(q R_{e q}\right)^{-x}$ excluding the Enhanced Backscattering.

5. $q R_{e q} \sim 2 k R_{e q}$ The Enhanced Backscattering regime often present for refractive indices $m$ significantly larger than one.

In the Power Law regime, when the phase shift parameter

$\rho=2 k R_{e q}|m-1|$

is less than 1, i.e. in the Rayleigh-Debye-Gans (RDG) limit, the power law exponent is given by the Porod exponent, which is $\left(2 D_{m}-D_{s}\right)$ [15], where $D_{m}$ and $D_{s}$ are the mass and surface fractal dimensions, respectively. The Porod exponent for nonfractal 3D shapes is $4\left(D_{m}=3, D_{s}=2\right)$, while for fractal aggregates it is given by their fractal dimension $D_{f}\left(D_{f}=D_{m}=D_{s}\right)$. As $\rho$ increases, spherical particles begin to develop two power law regimes, the first quasi-power law regime develops after the Guinier regime and goes as $(q R)^{-2}$ until $q R \approx 1.2 \rho$ where there is a cross over to the second power law of $(q R)^{-4}$. On the other hand dusts and small irregularly shaped particles exhibit a single power law, the magnitude of the exponent decreases with increasing $\rho$.

Further functionality with $\rho$ has been found in light scattered in the forward direction i.e. $\theta=0$ when the scattered intensity is normalized by the Rayleigh scattering cross section

$d C_{s c a} / d \Omega=R^{6} k^{4}\left|\frac{m^{2}-1}{m^{2}+2}\right|^{2}$

When $\rho<1$, the forward scattering is equal to the Rayleigh cross section. As $\rho$ becomes larger than 1 , the relative forward scattering decreases. When $\rho>10$, it decreases by a factor of $\sim 3 / \rho^{2}$ relative to the Rayleigh cross section [2]. It is important to realize that for all particle shapes that Q-space analysis has been applied to, the analysis finds quantifiable power laws and $\rho$ functionality which could not be uncovered when simply plotting scattered intensity vs. the scattering angle $\theta$. 


\section{Model and Numerical Method}

\subsection{Gaussian Random Spheres}

Figure 1 shows an example of GRSs which are characterized by three parameters: 1) $\sigma$, the relative standard deviation that describes the deviations from a perfect sphere in the radial direction; 2) $v$, the power law index of the covariance function which controls the number of bumps and dips in a tangential direction; and 3 ) $a$, the mean radius from which deviations occur and that sets the overall size of the particle[16]. The GRSs are based on a statistical model where different sets of random numbers are used to generate distinct shapes, and the three characterizing parameters describe statistically an ensemble of GRS. Ten random particles were generated for each size parameter studied with Q-space analysis with $\sigma=0.2, v=3$ and $a$ varied according to the size parameter desired. There has been an immense amount of work done in both describing GRSs and the light scattering from GRSs [10-13,16-24]. General descriptions of the GRS shape descriptions can be found in $[12,13,16]$. For a detailed mathematical description of GRSs the reader is directed to $[25]$.

\subsection{Discrete dipole approximation}

The discrete dipole approximation (DDA) is a method for calculating the scattering and absorption from an arbitrarily shaped particle. In DDA the particle volume is broken up into sub-volumes, each sub-volume is treated as a dipole [2628]. Numerical techniques are then used to calculate the polarization at each dipole due to the incident electric field and the scattered fields from all other dipoles. Once the polarizations of each dipole are known scattering quantities, such as the full scattering Mueller matrix, the total scattering and absorption can be calculated.

DDA was used to perform the light scattering calculation for the GRSs. Two separate DDA codes have been used. For the size parameters $k R_{e q}=$ $10,12,14,16,18,20$, a DDA code developed by our group was used [29,30]. For the largest size parameters of $k R_{e q}=30$, Amsterdam DDA (ADDA) method was used [28]. ADDA is a well established, documented and widely used code. To insure the validity of the code developed by our group, test cases of $k R_{e q}=10, m=1.2,1.3,1.5$ ran with both codes were compared and found to be in agreement. One of the large advantages of ADDA is its ability to run across multiple cores. Even with the multicore ability of ADDA these calculations can be very computationally costly especially as the particle size and index of refraction increase. To limit the computational costs of DDA, sets of ten shapes were chosen. For size parameters $k R_{e q}=$ $10,12,14,16,18,20,30$, the particles were described by an average of 142,000 ; 244,$000 ; 384,000 ; 569,000 ; 806,000 ; 1,098,000$; and 3,664,000 dipoles respectively with $\pm 10 \%$ deviation from particle to particle. Although there is a large range of the number of dipoles used to describe the different size parameters investigated, the number of dipoles per wavelength was kept constant at $\sim 18$.

DDA calculations were performed at forty random orientations per shape, the orientations are described by the three Euler angles $\alpha, \beta$, and $\gamma$ using the "zyznotation" [31,32]. For the Euler angles $\alpha$ and $\beta$ random angles between 0 and $2 \pi$ 
were used, values were randomly selected between -1 and 1 and used to calculate $\beta$ to avoid over selecting angles toward the poles. As a test to insure that an acceptable number of orientations were being used, a set of ten shapes was run at 40, 60 and 80 orientations per shape and then the results were averaged over the ten particles. Although there are some minor deviations at 80 orientations compared to 40 , the overall behavior of the scattered intensity vs. $q R_{v e q}$ is unaffected by orientations above 40 .

\section{Results and Discussion}

\subsection{Q-space analysis of GRS}

Figure 2 shows the orientationally averaged scattered light intensity for a set of ten GRSs calculated with the DDA, and normalized to unity at $q=0$ plotted versus $q R_{\text {veq. }}$. In this figure the effect of varying both $\sigma$ and $v$ using all possible permutations of $\sigma=0.1,0.2,0.3$ and $v=2,3,4$ at a size parameter of $k R_{v e q}=10$ and refractive index $m=1.3$ are considered and compared to the monodisperse scattering of spheres calculated using a Mie scattering code based on the code developed by Bohren and Huffman[33].

Figure 2 shows that variations in $v$ had a minimal overall effect on the light scattering as discerned by Q-space analysis. Figure 2 also shows that the lower the value of $\sigma$ the scattering by GRSs trend toward that of spheres, consistent with the fact that in the limit of $\sigma=0$ they become spheres. For $\sigma>0$, the orientational averaging effectively smooths the edges of the particle hence smoothing out the intensity ripples. Similar results have been reported in [16] where GRSs of $\sigma=0.1$ and 0.2 were compared to spheres. For $\sigma=0.1$ it was noted that the main deviation from that of spheres was in the backscattering. With $\sigma=0.2$, a smoothing of the ripples followed by some deviations in the backscattering was reported. The small effect of $v$ seen in Fig. 1 and small $\sigma$ being closely related to the solutions of spheres combined with the considerations of computational time limitations led us to a fixed choice of $\sigma=0.2$ and $v=3$ for the remainder of the study.

Figure 3 shows the orientationally averaged, forward -normalized scattered intensity as a function of $q R_{\text {veq }}$ for GRSs. This is essentially a plot of the phase function in q-space. The refractive index in these plots was varied to explore the behavior with changing phase shift parameter $\rho$. These plots exhibit the same qualitative features that have been previously seen for scattering by other types of shapes when Q-space analysis is applied (see Section 2 above) most notably power laws when $q R_{\text {veq }}>1$. As $\rho$ increases the power law exponents decrease similar to the dusts and irregular shapes that Q-space analysis has been applied to 
previously[7,8]. Finally, with increasing refractive index, an increasing development of the backscattering "the Glory" is seen.

Figure 4 shows the Rayleigh-normalized forward intensity vs. $\rho$ for both GRSs and spheres. When $\rho<1$, the GRSs scatter as Rayleigh particles; then as $\rho$ increases, the forward scattering decreases relative to Rayleigh scattering. It can be seen that the amount of decrease in the forward direction fluctuates slightly then settles down to a $3 / \rho^{2}$ functionality following closely with spheres.

In Fig. 5 the dependence of the scattering curves in each set have different size parameters and indices but the same $\rho$ values. As can be seen, the curves with the same $\rho$ values group together. Although not perfect, this quasi-universality with $\rho$ has been seen in other shapes.

\subsection{Comparison with Spherical Particle Mie Scattering}

In the following, we compare our results for the scattering of GRSs to the scattering by a polydisperse ensemble of spheres following a zeroth order log normal distribution (ZOLD) given by

$P(R)=\frac{\exp \left\{\frac{\left(-\left(\ln R / R_{0}\right)^{2}\right.}{2\left(\ln \sigma_{o}\right)^{2}}\right\}}{\sqrt{2 \pi} R_{0} \ln \sigma_{o} \exp \left\{1 / 2\left(\ln \sigma_{o}\right)^{2}\right\}}$

$\sigma_{o}$ in Eq. (4) is the deviation in the size distribution of spheres and $R_{0}$ is the most probable radius of the distribution[34]. In Fig. 6 the scattering of GRSs, is compared to the scattering by a polydisperse ensemble of spheres described earlier. When scattering for the polydisperse spheres with a twenty percent deviation is compared to that of the GRS with a twenty percent relative standard deviation, the light scattering curves from GRS and spheres follow along closely with each other except in the backscattering. As $\rho$ decreases toward the RDG limit of $\rho<1$, the scattered intensity of the spheres are slightly lower than that of the GRSs but continue to follow a similar trend to that of GRS with approximately equal value of power-law exponents. This interesting result shows that when orientationally averaged the scattered intensities from GRSs behave in a manner similar to that of a size distribution of spheres, smoothing out the ripple structure seen in monodisperse spheres. Yet the breaking of the spherical symmetry has significant impact, greatly reducing the enhanced backscattering.

\section{Conclusions}

The light scattering properties of GRSs were systematically studied with size parameters ranging from 10 to 30 , and with a relative index of refraction ranging from 1.01 to 1.5 . It was found that GRSs exhibit a q-independent forward scattering lobe when $q R_{\text {veq }}<1$, followed by the Guinier regime at $q R_{v e q} \approx 1$, and then a small dip in the light scattering between $2 \leq q R_{\text {veq }} \leq 5$. For larger $\mathrm{q} \mathrm{R}_{\text {veq }}$ a power law 
regime dominates with power law exponent that decreases with increasing phase shift parameter $\rho$. This behavior was quasi-universal with $\rho$, which is to say that the scattering curves were very similar when they had the same value of $\rho$ yet different size parameters and refractive indices. The Rayleigh normalized forward scattering intensity was unity for $\rho<1$ and then developed a $3 / \rho^{2}$ functionality for large $\rho$. Finally, as $\rho$ increased past 1, the GRSs exhibited an enhanced backscattering although not as significant as seen for spheres. We conclude that the behavior found for GRSs follows the general description for all shapes studied thus far, see Q-space summary 1-5, and exhibit power laws that behave similar to other perturbed spheres previously studied [8].

Single scattering studies done previously tend to focus on the scattered intensity vs. $\theta$. None have ever used Q-space analysis to study the scattered intensity to find distinct regimes (Rayleigh, Guinier, power law) in the scattered intensities of GRSs. Nor have the functionalities with the phase shift parameter been revealed in previous work. This work has continued the application of Q-space analysis to all types of particles. As with all shapes that Q-space has been applied to, we find quantifiable functionality with both $q R_{e q}$ and $\rho$ that would be difficult to uncover using traditional methods of plotting the scattered intensity vs. $\theta$.

\section{Acknowledgment}

This work was supported by the National Science Foundation, Directorate for Geosciences (GEO) (AGM 1261651); and the Army Research Office (ARO) (W911NF14-1-0352). We would like to thank Dr. Matthew Berg for allowing us to use his DDA code.

\section{References}

[1] Sorensen CM, Fischbach DJ. Patterns in Mie scattering. Opt Commun 2000;173:145-53. doi:10.1016/S0030-4018(99)00624-0.

[2] Berg MJ, Sorensen CM, Chakrabarti A. Patterns in Mie scattering: evolution when normalized by the Rayleigh cross section. Appl Opt 2005;44:7487-93. doi:10.1364/A0.44.007487.

[3] Xu M, Alfano RR. More on patterns in Mie scattering. Opt Commun 2003;226:15. doi:10.1016/j.optcom.2003.08.019.

[4] Berg MJ. Power-law patterns in electromagnetic scattering: A selected review and recent progress. J Quant Spectrosc Radiat Transf 2012;113:22-39. doi:10.1016/j.jqsrt.2012.05.015.

[5] Sorensen CM. Light scattering by fractal aggregates: A review. Aerosol Sci Technol 2001;35:648-87. doi:10.1080/02786820117868.

[6] Hubbard JA, Eckels SJ, Sorensen CM. Q-Space analysis applied to polydisperse, dense random aggregates. Part Part Syst Charact 2008;25:68-73. doi:10.1002/ppsc.200700014.

[7] Sorensen CM. Q-space analysis of scattering by dusts. J Quant Spectrosc Radiat Transf 2013;115:93-5. doi:10.1016/j.jpsrt.2012.09.001. 
[8] Sorensen CM, Zubko E, Heinson WR, Chakrabarti A. Q-space analysis of scattering by small irregular particles. J Quant Spectrosc Radiat Transf 2014;133:99-105. doi:10.1016/j.jqsrt.2013.07.020.

[9] Sorensen CM. Q-space analysis of scattering by particles: A review. J Quant Spectrosc Radiat Transf 2013;131:3-12. doi:10.1016/j.jqsrt.2012.12.029.

[10] Nousiainen T, Munoz O, Lindqvist H, Mauno P, Videen G. Light scattering by large Saharan dust particles: Comparison of modeling and experimental data for two samples. J Quant Spectrosc Radiat Transf 2011;112:420-33. doi:10.1016/j.jqsrt.2010.09.003.

[11] Munoz O, Volten H, Hovenier JW, Nousiainen T, Muinonen K, Guirado D, et al. Scattering matrix of large Saharan dust particles: Experiments and computations. J Geophys Res-Atmospheres 2007;112:D13215. doi:10.1029/2006JD008074.

[12] Veihelmann B, Nousiainen T, Kahnert M, van der Zande WJ. Light scattering by small feldspar particles simulated using the Gaussian random sphere geometry. J Quant Spectrosc Radiat Transf 2006;100:393-405. doi:10.1016/j.jqsrt.2005.11.053.

[13] Nousiainen T, Lindqvist H, McFarquhar GM, Um J. Small Irregular Ice Crystals in Tropical Cirrus. J Atmospheric Sci 2011;68:2614-27. doi:10.1175/2011JAS3733.1.

[14] Guinier A, Fournet G. Small-angle scattering of X-rays. Wiley; 1955.

[15] Porod G. Die Rontgenkleinwinkelstreuung Von Dichtgepackten Kolloiden Systemen .1. Kolloid-Z Z Polym 1951;124:83-114. doi:10.1007/BF01512792.

[16] Muinonen K, Zubko E, Tyynela J, Shkuratov YG, Videen G. Light scattering by Gaussian random particles with discrete-dipole approximation. J Quant Spectrosc Radiat Transf 2007;106:360-77. doi:10.1016/j.jqsrt.2007.01.049.

[17] Muinonen K, Pieniluoma T. Light scattering by Gaussian random ellipsoid particles: First results with discrete-dipole approximation. J Quant Spectrosc Radiat Transf 2011;112:1747-52. doi:10.1016/j.jqsrt.2011.02.013.

[18] Muinonen K. Light scattering by Gaussian random particles: Rayleigh and Rayleigh-Gans approximations. J Quant Spectrosc Radiat Transf 1996;55:60313. doi:10.1016/0022-4073(96)00004-0.

[19] Muinonen K. Introducing the Gaussian shape hypothesis for asteroids and comets. Astron Astrophys 1998;332:1087-98.

[20] Muinonen K, Lagerros JSV. Inversion of shape statistics for small solar system bodies. Astron Astrophys 1998;333:753-61.

[21] Zubko E, Muinonen K, Shkuratov Y, Videen G, Nousiainen T. Scattering of light by roughened Gaussian random particles. J Quant Spectrosc Radiat Transf 2007;106:604-15. doi:10.1016/j.jqsrt.2007.01.050.

[22] Lindqvist H, Muinonen K, Nousiainen T. Light scattering by coated Gaussian and aggregate particles. J Quant Spectrosc Radiat Transf 2009;110:1398-410. doi:10.1016/j.jqsrt.2009.01.015.

[23] Muinonen K, Nousiainen T, Lindqvist H, Munoz O, Videen G. Light scattering by Gaussian particles with internal inclusions and roughened surfaces using ray optics. J Quant Spectrosc Radiat Transf 2009;110:1628-39. doi:10.1016/j.jqsrt.2009.03.012. 
[24] Muinonen K. Introduction to light scattering by Gaussian random particles. J Quant Spectrosc Radiat Transf 2010;111:1745-7. doi:10.1016/j.jqsrt.2010.02.005.

[25] Lamberg L, Muinonen K, Ylonen J, Lumme K. Spectral estimation of Gaussian random circles and spheres. J Comput Appl Math 2001;136:109-21. doi:10.1016/S0377-0427(00)00578-1.

[26] Purcell E, Pennypac.cr. Scattering and Absorption of Light by Nonspherical Dielectric Grains. Astrophys J 1973;186:705-14. doi:10.1086/152538.

[27] Draine B, Flatau P. Discrete-Dipole Approximation for Scattering Calculations. J Opt Soc Am -Opt Image Sci Vis 1994;11:1491-9. doi:10.1364/JOSAA.11.001491.

[28] Yurkin MA, Hoekstra AG. The discrete-dipole-approximation code ADDA: Capabilities and known limitations. J Quant Spectrosc Radiat Transf 2011;112:2234-47. doi:10.1016/j.jqsrt.2011.01.031.

[29] Berg MJ, Sorensen CM, Chakrabarti A. Extinction and the optical theorem. Part II. Multiple particles. J Opt Soc Am -Opt Image Sci Vis 2008;25:1514-20. doi:10.1364/JOSAA.25.001514.

[30] Berg MJ, Sorensen CM. Internal fields of soot fractal aggregates. J Opt Soc Am Opt Image Sci Vis 2013;30:1947-55. doi:10.1364/JOSAA.30.001947.

[31] Mishchenko MI. Calculation of the Amplitude Matrix for a Nonspherical Particle in a Fixed Orientation. Appl Opt 2000;39:1026. doi:10.1364/A0.39.001026.

[32] Yurkin MA, Hoekstra AG. The discrete-dipole-approximation code ADDA: Capabilities and known limitations. J Quant Spectrosc Radiat Transf 2011;112:2234-47. doi:10.1016/j.jqsrt.2011.01.031.

[33] Bohren CF, Huffman DR. Appendix A: Homogeneous Sphere. Absorpt. Scatt. Light Small Part., Wiley-VCH Verlag GmbH; 1998, p. 477-82.

[34] Kerker M. CHAPTER 7 - Analysis of Particle Size. In: Kerker M, editor. Scatt. Light Electromagn. Radiat., vol. 16, Academic Press; 1969, p. 311-413. 


\section{Example of GRS $\sigma=0.2 v=3$}

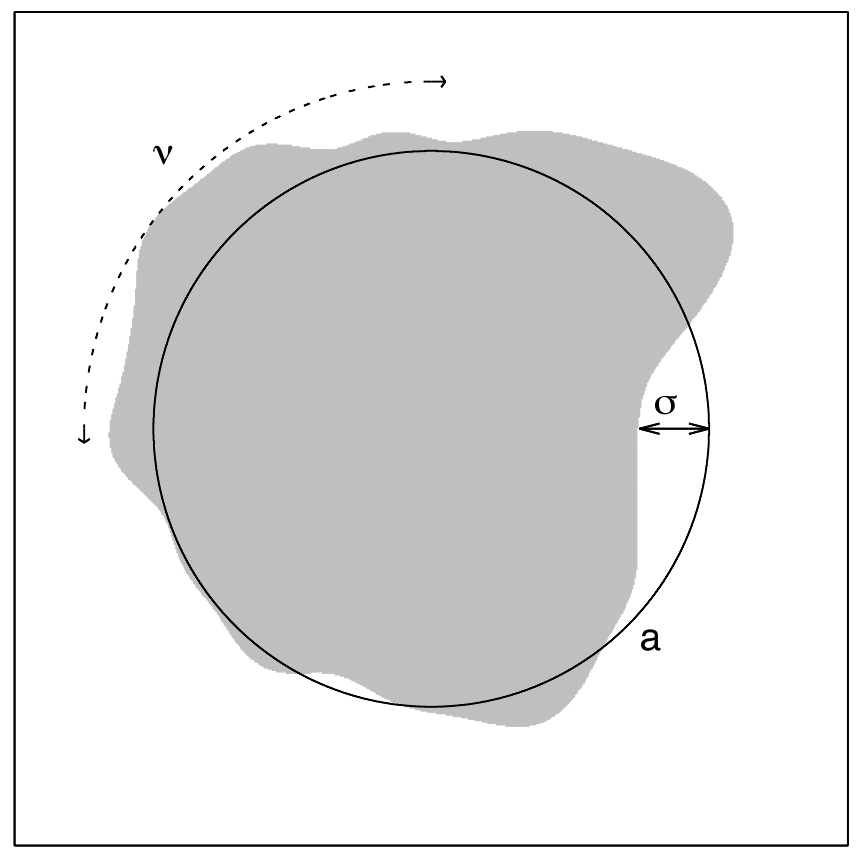

Fig. 1. Example of a GRS where $a$ is the mean radius, $\sigma$ the relative standard deviation controls the size of the fluctuations from the mean radius, and $v$ controls the number of fluctuations in an angular direction. 

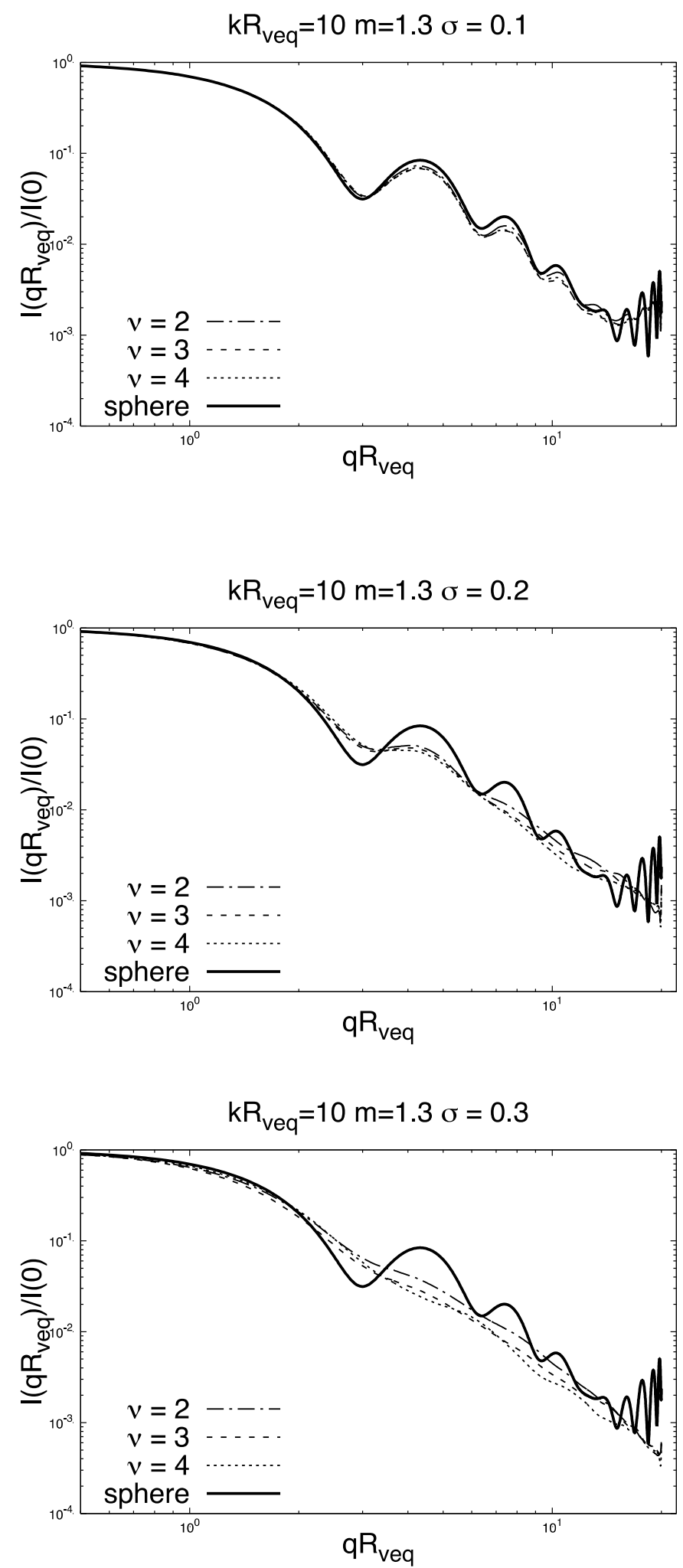

Fig. 2. Forward normalized scattered intensity of GRSs $k R_{\text {veq }}=10$ and $m=1.3$ for (top) $\sigma=0.1$ at $v=2,3,4$; (center) $\sigma=0.2$ at $v=2,3,4$; (bottom) $\sigma=0.3$ at $v=2,3,4$. 

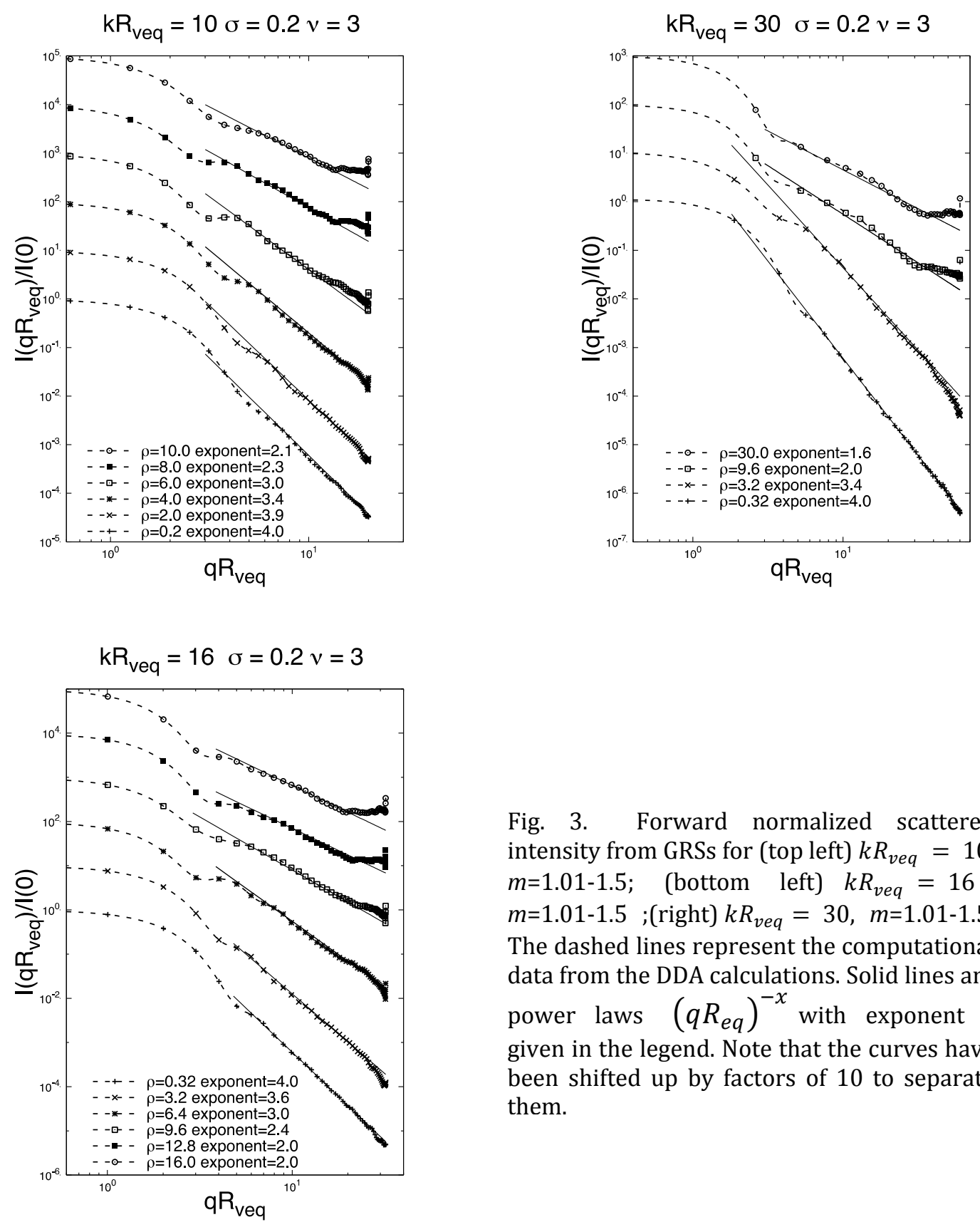

Fig. 3. Forward normalized scattered intensity from GRSs for (top left) $k R_{\text {veq }}=10$, $m=1.01-1.5 ; \quad$ (bottom left) $k R_{\text {veq }}=16$, $m=1.01-1.5$;(right) $k R_{\text {veq }}=30, m=1.01-1.5$. The dashed lines represent the computational data from the DDA calculations. Solid lines are power laws $\left(q R_{e q}\right)^{-x}$ with exponent $x$ given in the legend. Note that the curves have been shifted up by factors of 10 to separate them. 


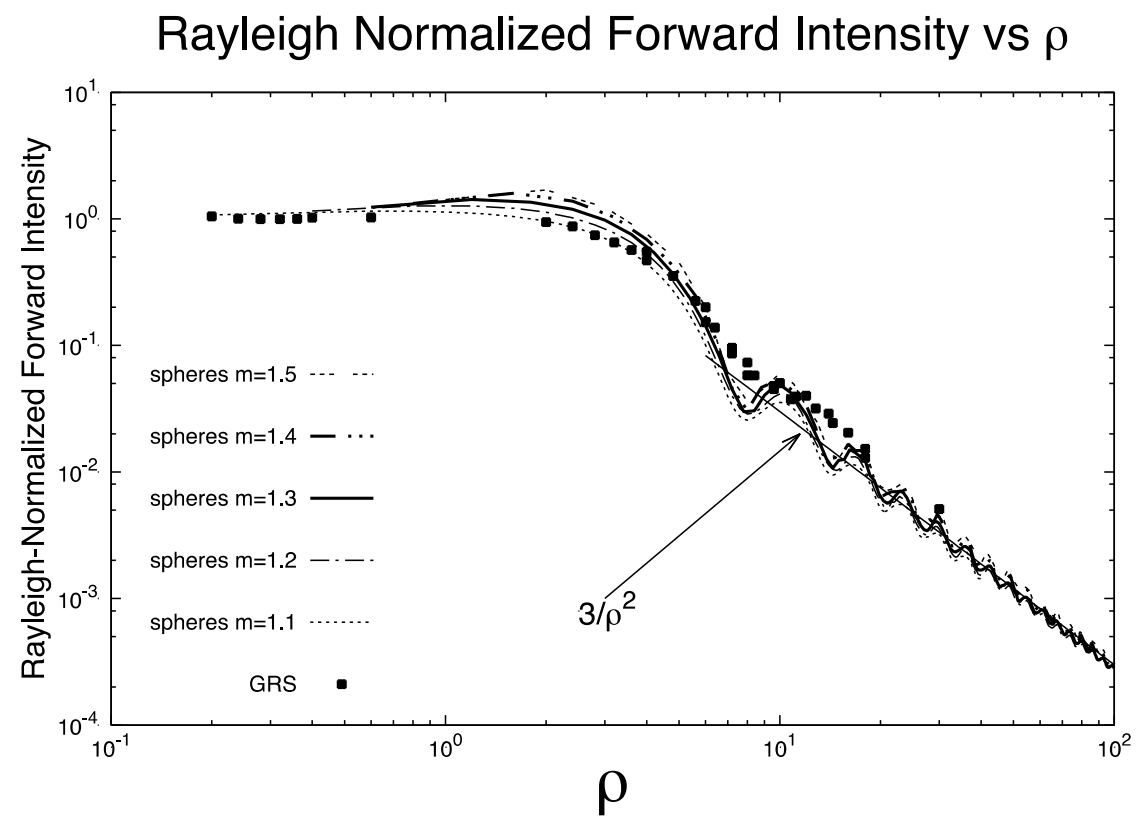

Fig. 4. Rayleigh Normalized forward intensity from GRSs and spheres with $m=$ 1.1-1.5 as a function of $\rho$. 


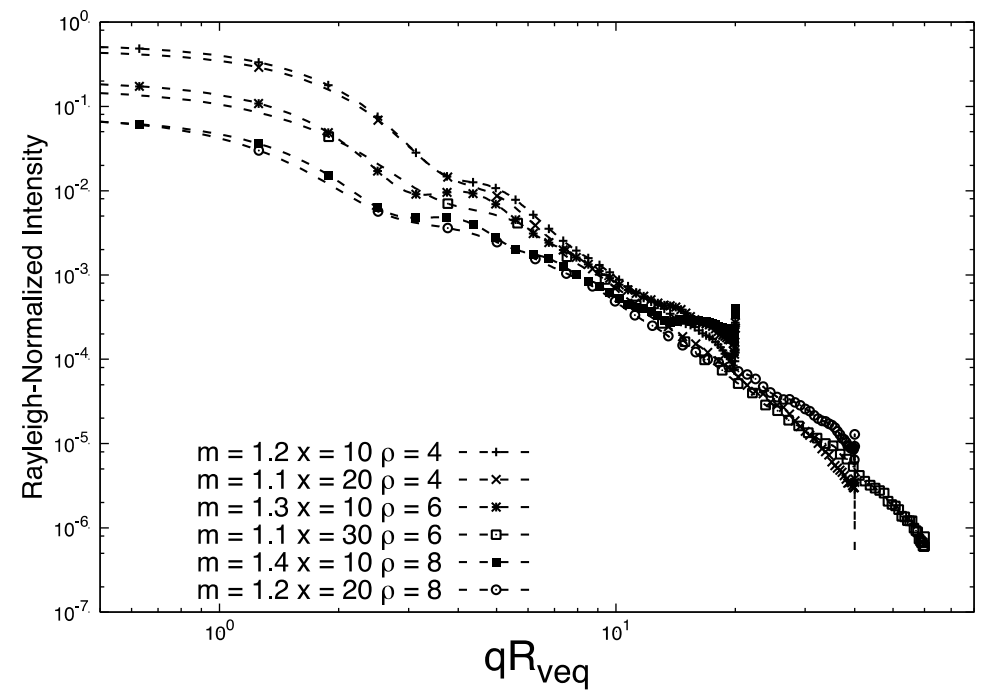

Fig. 5. Rayleigh normalized light scattering from GRSs with each set of curves having similar $\rho$ coming from distinct values of $k R_{\text {veq }}$ and $m$. 

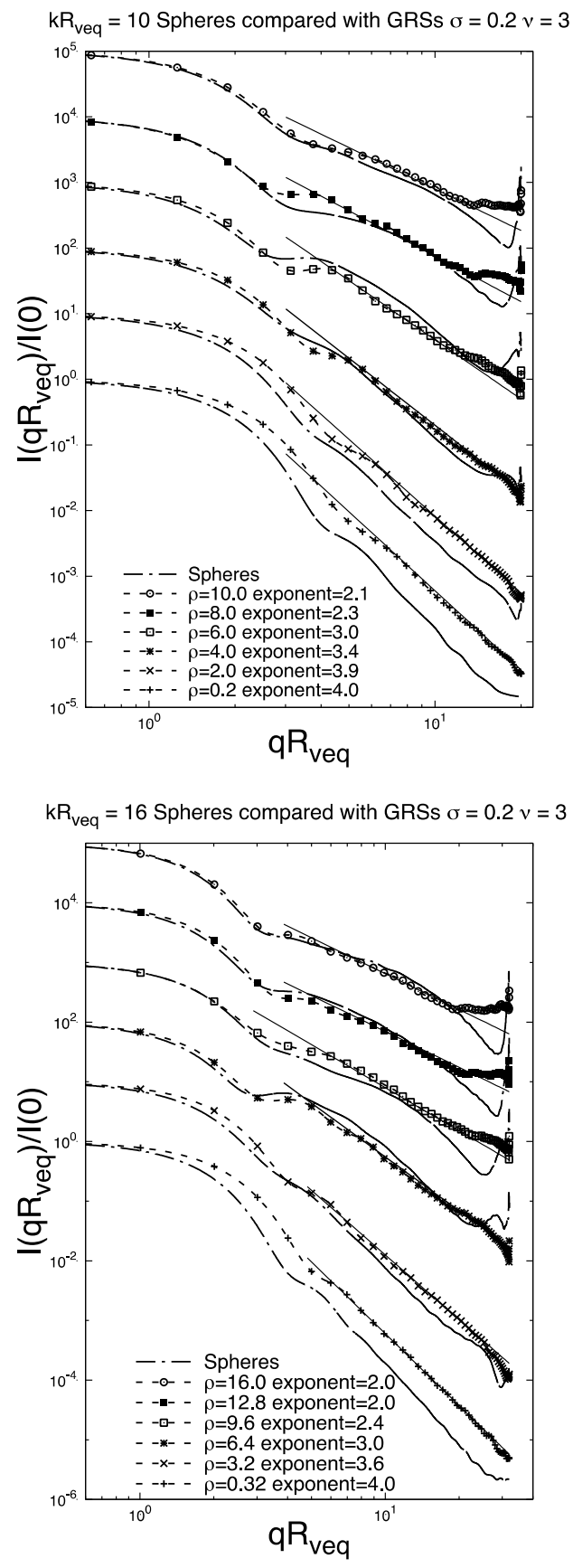

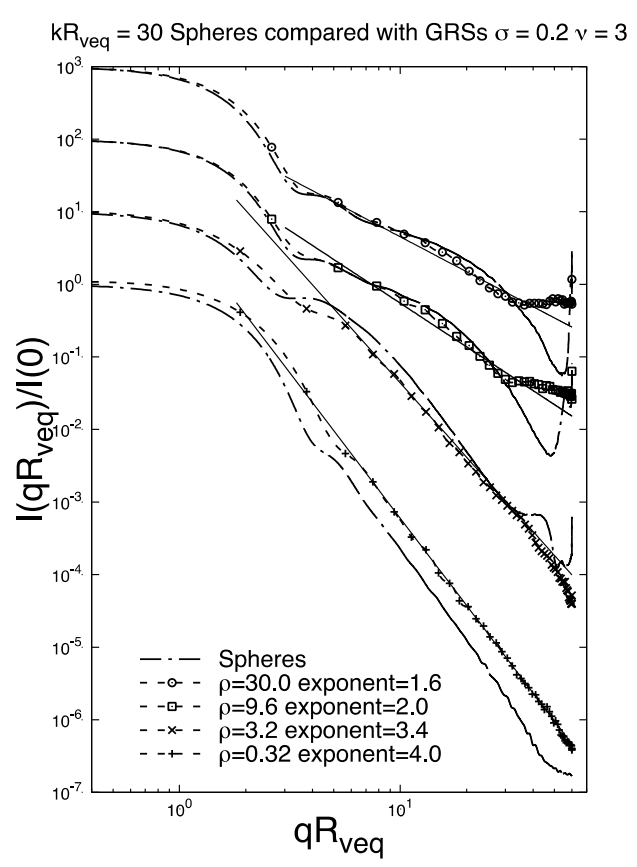

Fig. 6. Forward normalized scattered intensity of GRSs compared to spheres for (top left) $k R_{\text {veq }}=$ $10, m=1.01-1.5$; (bottom left) $k R_{v e q}=16$, $m=1.01-1.5$; (right) $k R_{\text {veq }}=30 \mathrm{~m}=1.01-1.5$. The dashed lines represent the computational data from the DDA calculations. Dash dot lines are Mie calculations for a size distribution of spheres with $\rho$ values of the most probable radius equivalent to the $\rho$ values of the GRSs shown, and a deviation in the distribution of radii of $\sigma_{o}=0.2$. Solid lines are power laws $\left(q R_{e q}\right)^{-x}$ with exponent $\mathrm{x}$ given in the legend. Note that the curves have been shifted up by factors of 10 to separate them. 